\title{
Visual system responds to rotational and size-change components of complex proximal motion patterns
}

\author{
MAURICE HERSHENSON \\ Brandeis University, Waltham, Massachusetts
}

\begin{abstract}
The qualitative and quantitative characteristics of rotation, contraction, and motion-in-depth motion aftereffects (MAEs) produced by rotating Archimedes spirals were compared for spirals differing in arm length $\left(90^{\circ}, 270^{\circ}\right.$, and $\left.720^{\circ}\right)$. There were three test stimuli. The spokes and rings test stimuli were figures whose contours were orthogonal to the direction of motion of the MAEs produced by the rotational and radial components of the spiral's motion, respectively. The floating disk test stimulus was used to test for a motion-in-depth MAE. In Experiment 1, naive subjects viewed the spirals for $30 \mathrm{sec}$ and $3 \mathrm{~min}$. The MAE components reported corresponded to the rotational and radial components of motion in the proximal stimulus. In Experiment 2, experienced subjects were used to measure decay time constants (DTCs) after $15 \mathrm{~min}$ of adaptation. The rotation MAE had a DTC that was shorter than the other two DTCs. Results support the hypothesis that the complex stimulus produced by rotating Archimedes spirals is resolved into rotational and radial components by structures in the visual system that are specifically sensitive to rotational and size-change relative motion patterns (Regan, 1986). They also support Hershenson's (1982) suggestion that the spiral aftereffect is produced by the same perceptual structures that mediate the perception of rigid object motion in depth.
\end{abstract}

Rotational and size-change motions are relational proximal stimulus motion patterns to which the human visual system may be specifically sensitive (Regan, 1986). The idea that the visual system contains "rotation detectors" is supported by evidence from studies of motion aftereffects (MAEs) and motion detection thresholds. For example, Cavanagh and Favreau (1980) used a rotating spiral to produce MAEs of different durations over test patterns that included the stationary adapting spiral as well as its mirror image, a figure whose contours were orthogonal to those of the adapting spiral. Cavanagh and Favreau argued that if the MAEs were mediated by units in the visual system sensitive only to linear motion, they should not have been observed over the test pattern whose contours were orthogonal to those of the adapting stimulus. Therefore, they proposed that the visual system contained structures that responded specifically to rotation in the proximal stimulus. Regan and Beverley (1985) compared motion detection thresholds produced by viewing a stimulus that had pure rotary motion after viewing a rotating or an equivalent linear motion pattern. The rotary adapting stimulus produced a greater elevation in the threshold for motion detection than did the equivalent linear adapting stimulus. Once again, the data supported the idea that the visual system contains units that are specifically sensitive to rotation in the proximal stimulus.

Beverley and Regan (1979) found evidence for structures sensitive to one-dimensional size change. They com-

I thank James P. Leahy for collecting the data. Please address correspondence to Maurice Hershenson, Department of Psychology, Brandeis University, Waltham, MA 02254. pared the MAEs produced by a bar of constant height whose vertical edges moved with a ramping motion that was either in phase or $180^{\circ}$ out of phase. When the vertical edges moved in phase, the bar moved laterally in the frontal plane and remained constant in size. When the vertical edges moved $180^{\circ}$ out of phase, the bar remained stationary but increased or decreased in size in its horizontal dimension. Beverley and Regan found that the MAE produced by the changing-size stimulus had a different decay time constant (DTC) than did the linear MAE produced by the same bar over the same retinal area. This difference in the storage property suggested to Beverley and Regan that the visual system contains units that are specifically sensitive to size change in the proximal stimulus.

Size change in two or more meridians produces an expansion or contraction pattern. Regan and Beverley (1978) found evidence for a structure specifically sensitive to expansion (looming) or contraction. They measured the relative amount of elevation in the threshold for detecting the oscillating motion of the sides of a small square after adapting to oscillating motions that were either in phase or $180^{\circ}$ out of phase. When the opposite sides of the adapting square oscillated in phase, the square maintained a constant size but moved back and forth along a diagonal. When the opposite sides of the square oscillated $180^{\circ}$ out of phase, the square remained stationary but increased and decreased in size. The relative amounts by which the thresholds were elevated differed markedly for the two cases; that is, the differential adaptation was specific to the relationship between the motion of the opposite edges 
of the square rather than to the local motion of any given edge.

The suggestion that the visual system is specifically sensitive to expansion and contraction patterns in the proximal stimulus has direct relevance for theories of visual space perception, because this is the proximal pattern produced by rigid distal objects moving directly toward or away from the observer. Indeed, in Regan and Beverley's (1978) experiment, the expanding and contracting square appeared to be moving in depth rather than changing in size. This relationship has been emphasized by Hershenson $(1982,1984)$, who noted that the spiral aftereffect (SAE) consists of perceived motion in depth as well as frontal plane expansion or contraction and, therefore, suggested that the SAE and the perception of motion in depth are mediated by the same structures in the visual system.

If this view of the visual system is correct, one would expect that the structures in the visual system would respond in their characteristic ways when stimulated by complex proximal stimulus motions that contain combinations of the simple motions to which the structures are specifically sensitive. Thus if the visual system were stimulated by a complex proximal motion pattern, the rotation detectors should respond to the rotational component of the complex motion and the size-change detectors should respond to the size-change components of the pattern. Furthermore, if the proximal stimulus contained an expansion or contraction component (i.e., size change in two or more meridians), then the expansion/contraction detectors should respond to this component of the proximal stimulus. The differential sensitivities of these visual system structures should be manifested in the MAEs observed over test stimuli orthogonal to the component of motion in the proximal stimulus to which the detector is sensitive.

The experiments reported here tested these ideas directly by using rotating Archimedes spirals as adapting stimuli. Archimedes spirals were selected because the motion of any point on a spiral can be described by a vector normal to the contour at that point. This vector is readily resolved into rotational and radial motion components. Moreover, the relative strength of these components can be varied by using spirals of different arm lengths (Scott \& Noland, 1965). For example, when rotated, short spirals (approximately $90^{\circ}$ or $1 / 4$ turn) produce motion patterns that have relatively large rotational components, whereas longer spirals produce motion patterns that have relatively large radial components.

This paper describes the qualitative and quantitative characteristics of the rotation, contraction, and motionin-depth MAEs produced by rotating Archimedes spirals of three different arm lengths: $90^{\circ}, 270^{\circ}$, and $720^{\circ}$. Figure 1 shows the motion vector profiles for the three spirals. Each graph depicts the relative sizes of the normal, radial, and rotational motion vectors as a function of distance from the center of rotation. The $90^{\circ}$ spiral has a relatively strong rotational component over the entire length of the spiral. The radial component is relatively weak near the center of rotation, equal to the rotational component about halfway out toward the edge of the disk, and relatively strong thereafter. The $270^{\circ}$ spiral has a relatively strong radial component and a weaker but not inconsequential rotational component throughout its length. The $720^{\circ}$ spiral has a relatively very strong radial component and a relatively very weak rotational component over the entire length of the spiral.

\section{GENERAL METHOD}

\section{Adapting Stimuli}

The three adapting stimuli were left-throw Archimedes spirals whose arm lengths were $90^{\circ}, 270^{\circ}$, and $720^{\circ}$ of arc. The arms were drawn in black ink and were approximately $1.5 \mathrm{~cm}$ wide. The spirals subtended approximately $3.3^{\circ}$ of visual angle. They rotated in a clockwise direction at $60 \mathrm{rpm}$.

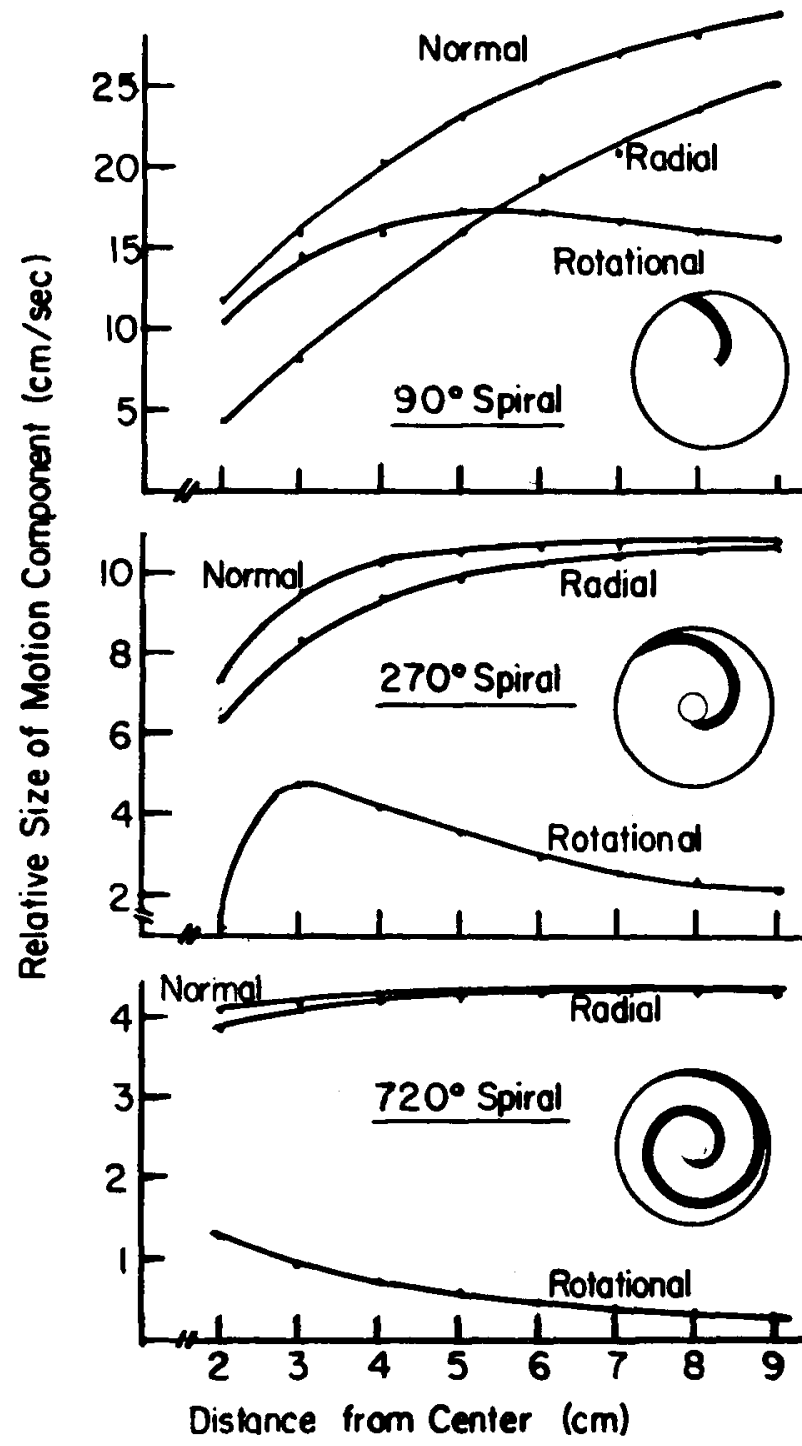

Figure 1. Motion vector profiles for the three spirals, $90^{\circ}$ $(e=5.52 \theta), 270^{\circ}(\varrho=1.76 \theta)$, and $720^{\circ}(\varrho=.69 \theta)$, showing the relative size of normal, radial, and rotational motion vectors as a function of distance from the center of rotation. 


\section{Test Stimuli}

The three types of MAEs were measured separately, using test stimuli whose contours were orthogonal to the direction of motion of the MAE component for radial and rotational MAEs and using a floating disk for the motion-in-depth aftereffect. The spokes test stimulus consisted of four equally spaced diameters produced by black lines of 2-mm thickness in an imaginary circle on white paper. It subtended approximately $3.3^{\circ}$ of visual angle. The rings test stimulus consisted of five concentric equally spaced black circles $2 \mathrm{~mm}$ thick drawn on white paper. The largest circle subtended slightly less than $3.3^{\circ}$ of visual angle. These test stimuli were mounted on a white background that was the same distance from the viewer as the adapting stimulus. The black floating disk test stimulus was suspended by two thin white crossed strings in the test area, approximately $18 \mathrm{~cm}$ in front of this white background. It subtended approximately $1.5^{\circ}$ of visual angle and appeared to float in space with little or no support.

\section{Procedure}

The subject's head was held relatively immobile in a chin-andhead holder. The adapting stimulus was viewed through an aperture measuring $4^{\circ}$ of visual angle horizontally and $6^{\circ}$ of visual angle vertically. The occluding panel contained a sliding door that exposed the adapting stimulus field when in one position and the test field when in the other position. The subject fixated a small red circle at the center of rotation of the adapting spiral with his/her right eye and, when the signal was given, shifted fixation to the center of the test stimulus. The shift was accomplished in less than $1 / 4 \mathrm{sec}$. The adapting and test fields were illuminated by two $75-\mathrm{W}$ floodlights, approximately $1.8 \mathrm{~cm}$ from the plane of stimulation.

The subject was told to fixate the center of the test stimulus. If he/she saw motion in the aftereffect, he/she was to rate the strength of motion on an 11-point scale, on which 10 represented motion as strong as that of the rotating spiral (in Experiment 1) or the initial speed of perceived motion (in Experiment 2) and 0 represented no motion. Immediately upon shifting fixation to the test stimulus, the subject began calling out rating numbers and continued until zero motion was reported twice in a row. Judgments were recorded every $5 \mathrm{sec}$. After each trial, the subject described the perceived motion in his/her own words.

\section{EXPERIMENT 1}

\section{Method}

Two adaptation times, $30 \mathrm{sec}$ and $3 \mathrm{~min}$, and three arm lengths were used for each of the three test stimuli. The presentation order of adaptation and test stimulus combinations was governed by a Latin square. There were six repetitions of each adaptation-test stimulus combination for each spiral.

The subjects were 5 undergraduates who did not know the purpose of the experiment. None had previously observed MAEs or participated in a perceptual experiment.

\section{Results and Discussion}

Qualitative reports. The qualitative differences in the MAEs produced by the three spirals are striking. The $90^{\circ}$ spiral produced a MAE only when the spokes test stimulus was used. It was described by all subjects as perceived rotation of the spokes in a direction opposite that of the spiral. In contrast, the $270^{\circ}$ and $720^{\circ}$ spirals produced MAEs primarily when the rings and floating disk were the test stimuli ( 1 subject did not report a MAE over the rings test stimulus after viewing the $270^{\circ}$ spiral for $30 \mathrm{sec}$ ). Subjects described the MAE observed over the rings test stimulus as contraction of the rings in the frontal plane. After viewing the floating disk test stimulus, they reported that the disk appeared to move in depth, directly away from them.

The rotating $90^{\circ}$ spiral produced a proximal stimulus pattern with a relatively large rotational component, whereas the longer spirals produced patterns with relatively large radial components. The fact that the $90^{\circ}$ spiral produced a strong rotation MAE and the other spirals produced strong contraction MAEs supports the idea that the visual system contains units that respond specifically to these components of motion in the proximal stimulus. The fact that the spirals with the large radial components produced both a strong contraction MAE and a strong motion-in-depth MAE supports Hershenson's (1982) proposal that the SAE is mediated by the same structures in the visual system that mediate the perception of motion in depth. Moreover, both findings support the hypothesis that the visual system is structured in such a way as to process relative motion in the proximal stimulus ( $\mathrm{Re}-$ gan, 1986), that is, "global" input from different parts of the visual field.

The rotating $90^{\circ} \mathrm{spiral}$ also produced a stimulus with a radial component that was relatively strong over the outer portion of the disk, but it did not produce either a contraction or a motion-in-depth MAE. This finding also supports the structural hypothesis of Regan and Beverley (1978; Beverly \& Regan, 1979), because they described units that respond to size change in one or more meridians. A radial vector is only one half of the size-change stimulus pattern required to activate such a structure. Both the $270^{\circ}$ and $720^{\circ}$ spirals produce nearly simultaneous radial vectors that are $180^{\circ}$ out of phase; the $90^{\circ} \mathrm{spiral}$ does not. Therefore, it would not be expected that this stimulus would activate a size-change structure.

Quantitative differences. The quantitative properties of the MAEs were indexed by their DTCs, calculated for each adaptation time $\times$ arm length $\times$ test stimulus combination for each subject. Because the log of the strength ratings decayed linearly over time, the line of best fit was computed based on the six within-cell repetitions. The DTC was defined as the time it took for the strength of the MAE to drop to $1 / e$ (approximately .368) of its initial value, using the line of best fit as a model of the decay.

The average DTCs were 17.4, 17.3, and $20.7 \mathrm{sec}$ for the rotational, contraction, and motion-in-depth MAEs, respectively. Because of the many empty cells, an ANOVA was computed using the DTCs produced ony by the $270^{\circ}$ and $720^{\circ}$ spirals when the rings and floating disc were the test stimuli. ${ }^{1}$ None of the interactions were significant. The difference between the contraction and motion-in-depth DTCs was significant $[F(1,4)=27.40$, $p<.01]$. The longer spiral produced slightly longer average DTCs: $19.9 \mathrm{sec}$ for the $720^{\circ}$ spiral and $18.0 \mathrm{sec}$ for the $270^{\circ}$ spiral $[F(1,4)=8.61, p<.05]$. The $90^{\circ}$ spiral produced an average DTC of $17.4 \mathrm{sec}$. For the two longer spirals, the 3-min adaptation time produced MAEs with an average DTC of $21.5 \mathrm{sec}$, and the $30-\mathrm{sec}$ adaptation 
time produced MAEs with an average DTC of $16.4 \mathrm{sec}$ $[F(1,4)=14.99, p<.05]$. This difference was also manifested in the MAEs produced by the $90^{\circ}$ spiral: the 3-min adaptation time produced MAEs with an average DTC of $19.8 \mathrm{sec}$, and the 30-sec adaptation time produced MAEs with an average DTC of $15.0 \mathrm{sec}$.

The DTCs for the contraction and motion-in-depth MAEs differed only by $3.4 \mathrm{sec}$. Although this difference was significant in the analysis, it may be an artifact of the differences in the test situations and of the difficulty the inexperienced subjects had in making the ratings in the two situations. Moreover, the theoretical expectation was that these two MAEs should not have different DTCs because they are mediated by the same underlying structure in the perceptual system. The expected difference was between these two and the rotation MAEs, but there was hardly any difference between them. Consequently, it is probably best not to use the quantitative data from inexperienced subjects as evidence in evaluating the hypotheses about the possible existence of structures in the visual system. In Experiment 2, experienced subjects were used to shed light on this issue by comparing more stable DTCs obtained with long adaptation times.

\section{EXPERIMENT 2}

In this experiment, the $270^{\circ}$ spiral was used because it produced a strong radial component and a weaker but not insignificant rotational component of motion. It was expected, therefore, that this stimulus would permit withinsubject comparisons of the three different MAEs produced by a single adapting stimulus. The $720^{\circ}$ spiral was used to permit comparison of the MAEs produced by radial components of motion that differed in strength. If the visual system responds to these components, the difference in strength of the components should produce measurable differences in the MAEs produced by the radial components of the two different complex motion patterns.

\section{Method}

Subjects adapted for $15 \mathrm{~min}$ to the $270^{\circ}$ and $720^{\circ}$ spirals. Presentation order of adaptation stimuli followed a Latin square. There were three repetitions of each adaptation-test stimulus combination. Test stimuli were presented in blocks in three separate sessions, counterbalanced across subjects.

The subjects were 4 undergraduate students who had experience in rating the decay of MAEs.

\section{Results and Discussion}

All subjects reported contraction and motion-in-depth MAEs over the rings and disk test stimuli, respectively. A rotation MAE was reported by 3 of the 4 subjects for the spokes test stimulus after adapting to the $270^{\circ}$ spiral. None reported a rotation MAE for the $720^{\circ}$ spiral.

Three different MAEs were produced by the same adapting stimulus for the $270^{\circ}$ spiral. An ANOVA was performed on the DTCs produced by the 3 subjects who reported rotation MAEs after viewing the $270^{\circ}$ spiral stimulus. There were three replications of each test stimu- lus for each subject. The main effect of test stimulus was significant $[F(2,4)=19.39, p<.01]$. The prediction that the average DTC for the rotation MAE $(6.5 \mathrm{sec})$ differs from the average DTC for the radial $(14.9 \mathrm{sec})$ and the motion-in-depth $(14.8 \mathrm{sec})$ MAEs was supported $[F(1,4)$ $=38.78, p<.01]$. The fact that the rotation MAE produced by the $270^{\circ}$ spiral had a shorter DTC than either the radial or the motion-in-depth MAE suggests that rotation MAEs are mediated by a structure or structures that are different from those that mediate the other two MAEs. Whether these structures are different from simple motion detectors in that they respond to the global rotational stimulus cannot be determined from this experiment.

The radial and motion-in-depth MAEs were compared using the data from all 4 subjects. An ANOVA was performed on the DTCs on three replications of each adaptation $\times$ test stimulus cell for each subject. The interaction of arm length with test stimulus (radial $=15.2 \mathrm{sec}$ and motion in depth $=14.0 \mathrm{sec}$ for the $270^{\circ}$ spiral; radial $=23.0 \mathrm{sec}$ and motion in depth $=24.9 \mathrm{sec}$ for the $720^{\circ}$ spiral) was significant $[F(1,4)=36.78, p<.01]$. The difference due to type of test stimulus (MAE) was small: $19.1 \mathrm{sec}$ for rings (contraction MAE) and $19.4 \mathrm{sec}$ for the floating disk (motion-in-depth MAE) and was not significant $[F(1,4)=.009, p>.05]$.

The fact that the $720^{\circ}$ spiral produced a longer lasting radial and motion-in-depth MAE than did the $270^{\circ}$ spiral supports the contention that these MAEs were produced by the radial component of the motion of the spirals. Along with the fact that the DTCs measured over the rings and disk test stimuli were almost exactly the same for each spiral, the results support the hypothesis that the same structure in the visual system mediates the contraction component of the SAE and the perception of motion in depth (Hershenson, 1982).

\section{GENERAL DISCUSSION}

The major finding is that the visual system responds to the rotational and radial components of the complex motion patterns produced by the rotating Archimedes spirals. The $90^{\circ}$ spiral with a large rotational component produced a strong rotational MAE over the spokes test stimulus; the $270^{\circ}$ spiral with a small but significant rotational component produced a weak rotational MAE that was reported by 3 of the 4 experienced observers. Strong contraction MAEs were reported over the rings test stimulus for both the $270^{\circ}$ and $720^{\circ}$ spirals, both of which produced large radial motion components. These qualitative observations support the notion that the visual system contains structures that are organized to respond selectively to radial and rotational motion vectors (Beverley \& Regan, 1979; Cavanaugh \& Favreau, 1980; Regan, 1986; Regan \& Beverly, 1978).

Strong motion-in-depth MAEs were reported over the floating disk whenever strong contraction MAEs were reported over the rings, an observation that supports the hypothesis that the radial component of the complex mo- 
tion patterns initated both responses. It suggests, in addition, that these two MAEs were mediated by the same underlying structures in the visual system. This conclusion is also supported by the quantitative findings: the DTCs for these qualitatively different MAEs were almost identical. Furthermore, the data support the hypothesis that these MAE components of the SAE are mediated by the same structure in the perceptual system that mediates the perception of motion in depth (Hershenson, 1982).

The quantitative evidence also indicates that different structures were responding to the rotational and radial motion components to produce the rotational and contraction MAEs. The DTC for the rotational MAE was significantly shorter than that for the contraction MAE. Whether the rotational MAE is produced by a structure in the visual system that is different from simple motion detectors is not clear, because the DTCs produced by the experienced viewers to rotation components of the spirals were well within the range reported for simple linear MAEs (Regan \& Beverley, 1985). It is not possible, therefore, to answer this question with these stimuli alone. Nevertheless, it is possible to say with some certainty that the structure in the visual system that mediates the contraction and motion-in-depth MAEs is different from the structure that mediates the rotation MAE.

In summary, the complex stimulus produced by rotating Archimedes spirals is resolved into rotational and radial components by the structures that make up the visual system. The response of these structures is manifested not only in the rotational and contraction MAEs, but also in the perception of motion in depth.

\section{REFERENCES}

BeverLey, K. I., \& RegaN, D. (1979). Separable aftereffects for changing size and motion in depth: Different neural mechanisms. Vision Research, 19, 727-732.

Cavanagh, P., Favreau, O. E. (1980). Motion aftereffect: A global mechanism for the perception of rotation. Perception, 9, 175-182.

Hershenson, M. (1982). Moon illusion and spiral aftereffect: Illusions due to the loom-zoom system? Joumal of Experimental Psychology: General, 111, 423-440.

Hershenson, M. (1984). Phantom spiral aftereffect: Evidence for global mechanisms in perception. Bulletin of the Psychonomic Society, 22, 535-537.

REGAN, D. (1986). Visual processing of four kinds of relative motion. Vision Research, 26, 127-145.

Regan, D., Beverley, K. I. (1978). Looming detectors in the human visual pathway. Vision Research, 19, 415-421.

Regan, D., Beverley, K. I. (1985). Visual response to vorticity and the neural analysis of optic flow. Joumal of the Optical Society of America, 2, 280-283.

SCOTT, T. R., Noland, J. H. (1965). Some stimulus dimensions of rotating spirals. Psychological Review, 72, 344-357.

\section{NOTE}

The only empty cell in this array was the $30-\sec 270^{\circ}$-rings cell for Subject 4. For purposes of the analysis, an estimated value was obtained by calculating this subject's average performance for the $30-\mathrm{sec}$ stimulus duration and weighting it in the same way as the corresponding cell at 3-min stimulus duration. This neutral value also entered into the averages. 